\title{
Nonlinear Squeezing for Measurement-Based Non-Gaussian Operations in Time Domain
}

\author{
Shunya Konno $\odot,{ }^{1}$ Atsushi Sakaguchi $\odot,{ }^{1}$ Warit Asavanant๑, ${ }^{1}$ Hisashi Ogawa $\odot,{ }_{1}^{1}$ Masaya Kobayashi, ${ }^{1}$ \\ Petr Marek $\odot,{ }^{2}$ Radim Filip, ${ }^{2}$ Jun-ichi Yoshikawa, ${ }^{1}$ and Akira Furusawa ${ }^{1, *}$ \\ ${ }^{1}$ Department of Applied Physics, School of Engineering, The University of Tokyo, 7-3-1 Hongo, Bunkyo-ku, \\ Tokyo 113-8656, Japan \\ ${ }^{2}$ Department of Optics, Palacký University, 17 listopadu 1192/12, Olomouc 77146, Czech Republic
}

(Received 30 November 2020; accepted 19 January 2021; published 10 February 2021)

\begin{abstract}
Quantum non-Gaussian gate is a missing piece to the realization of continuous-variable universal quantum operations in an optical system. In a measurement-based implementation of the cubic phase gate, a lowest-order non-Gaussian gate, non-Gaussian ancillary states that have a property we call nonlinear squeezing are required. This property, however, has never been experimentally verified. In this paper, we generate a superposition between a vacuum state and a single-photon state whose nonlinear squeezing is maximized by the optimization of the superposition coefficients. The nonlinear squeezing is observed via real-time quadrature measurements, meaning that the generated states are compatible with real-time feedforward and are suitable as ancillary states for the cubic phase gate in the time domain. Moreover, by increasing the number of photons, it is expected that nonlinear squeezing can be further improved. The idea presented here can be readily extended to higher-order phase gates [P. Marek et al., Phys. Rev. A 97, 022329 (2018)]. As such, this work presents an important step to extend continuous-variable quantum information processing from Gaussian regime to non-Gaussian regime.
\end{abstract}

DOI: 10.1103/PhysRevApplied.15.024024

\section{INTRODUCTION}

Continuous-variable (CV) quantum computation using optical systems is currently one of the most promising approaches to a scalable and practical quantum computation. As recent progress, large-scale Gaussian cluster states, the computational resource states for measurementbased quantum computation [1,2], have been experimentally realized using the time-domain-multiplexing method [3-6]. By implementing appropriate measurements on the Gaussian cluster states, universal CV quantum operations can be realized. Even more recently, by combining basis-programmable homodyne measurements with timedomain cluster states, Gaussian operations, i.e., linear transformations of the quadrature operators, have been demonstrated $[7,8]$. These experimental results demonstrate the potentials of the $\mathrm{CV}$ optical systems for quantum computation.

\footnotetext{
*akiraf@ap.t.u-tokyo.ac.jp
}

Published by the American Physical Society under the terms of the Creative Commons Attribution 4.0 International license. Further distribution of this work must maintain attribution to the author(s) and the published article's title, journal citation, and DOI.
It is known, however, that in addition to the Gaussian operations, at least a single non-Gaussian operation is required to achieve universality [9]. Moreover, because CV quantum computation that has no non-Gaussian elements can be effectively simulated with classical computation [10], a non-Gaussian element is a necessary requirement to achieve a useful quantum computation. One of the methods to realize non-Gaussian elements in measurement-based quantum computation is by implementing nonlinear measurements, i.e., measurements that are nonlinear in quadrature basis $[2,11]$. Note that since homodyne measurements are linear in the quadrature operators, direct nonlinear measurements would require some sort of additional inline strong optical nonlinearity, which is very difficult to realize. Luckily, there exists a feasible alternative: nonlinear quadrature measurements can be implemented using ancillary states, homodyne measurements, and nonlinear feedforwards based on the measurement results [11,12]. The implementations of the cubic phase gate (Fig. 1) and its generalization, $N$ th-order phase gates, based on this approach have been proposed $[13,14]$. Some progresses and basic prototypes have already been made regarding the nonlinear feedforward in the time domain $[15,16]$. Therefore, the next experimental hurdle that we must overcome is the generation of the non-Gaussian ancillary states. 


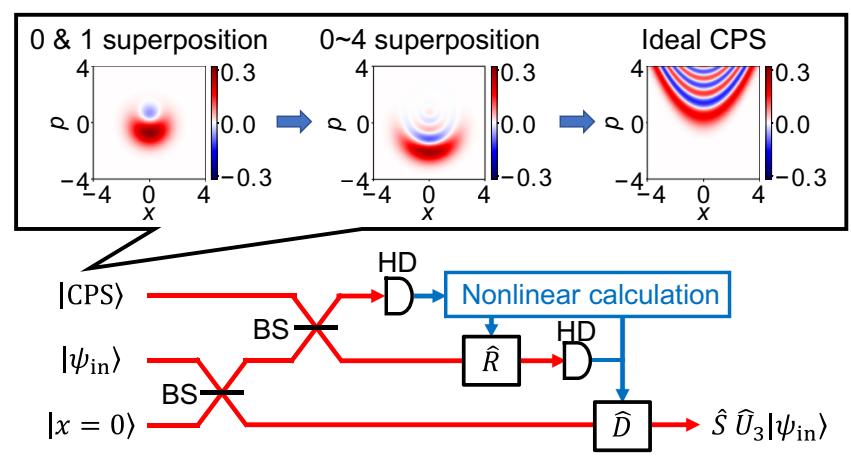

FIG. 1. Schematic diagram of implementation of cubic phase gate using non-Gaussian ancillary states, homodyne measurements, and nonlinear feedforwards. $|\mathrm{CPS}\rangle$, cubic phase state; BS, beam splitter; HD, homodyne detector; $\hat{R}$, phase rotation; $\hat{D}$, displacement operation; $\hat{S}$, squeezing operation; $\hat{U}_{3}$, cubic phase gate. The output of this setup is a cubic phase gate followed by a squeezing operation. Because the ideal CPS is unphysical, we need to consider approximations using the superpositions of Fock states. As the maximum photon number is increased, the nonlinear squeezing also approaches the ideal limit. Note that we omit displacement in the $p$ direction of the ancillary states.

As nonlinear feedforward operations introduce noises that are nonlinear in quadrature operators, the ideal ancillary states must be non-Gaussian states that are tailored to suppressing such noises. For example, an ancillary state of the cubic phase gate must suppress the noise with a form of $\hat{p}-3 \kappa \hat{x}^{2}$, where $\kappa$ is a real number. This idea is similar to how the amount of squeezing in the cluster states determines the amount of noise added when they are used as resources for Gaussian operations [17]. Therefore, we will call the suppression of the nonlinear noise in these non-Gaussian ancillary states nonlinear squeezing (NLSQ). Note that the squeezing of the nonlinear operators can be found in various non-Gaussian states. For example, Fock states have infinite squeezing regarding the operator $\hat{x}^{2}+\hat{p}^{2}$. However, Fock states are not suitable for suppressing noise in the cubic phase gate. In addition to high NLSQ, the ancillary states for nonlinear measurements should be compatible with the time-domainmultiplexing method. In time-domain $\mathrm{CV}$ quantum computation, modes are defined as localized temporal wave packets [18]. Therefore, to obtain the quadrature values, we need to integrate the signals from continuous homodyne measurements with the shape of the temporal modes. For general temporal modes, this integration introduces additional latency as an analog-to-digital (A/D) converter is required in the implementation of the digital integration. Latency can easily result in the decoherence of the system and has been one of the main technical difficulties of the measurement-based non-Gaussian gates that operate in the time domain. This difficulty can be overcome by engineering the shape of the wave packet of the ancillary states so that we can implement real-time quadrature measurements. In real-time measurement, the integration process can be replaced with a passive low-pass filter where the latency becomes simply the propagation time through the filter [19], which could be smaller than a nanosecond. This is at least an order of magnitude smaller than the latency of a typical A/D converter and digital integration. Experimentally, such real-time measurements have been demonstrated on single-photon states [19] and cat states [20]. However, generation of ancillary states that possess both NLSQ and compatibility with real-time measurements has not been achieved yet, as the NLSQ requires more optimization than the aforementioned quantum states. As a side note, this technical difficulty does not arise in linear feedforward because it is commutable with the integrations with the temporal modes.

In this paper, we experimentally generate quantum states that exhibit NLSQ necessary for the implementation of the cubic phase gate [13]. In the ideal limit, this state is called the cubic phase state and is infinitely nonlinearly squeezed. In general, achieving perfect NLSQ requires ancillary states that are unphysical, similar to how the generation of an ideal cluster state requires infinitely squeezed states, which are also unphysical. As an alternative, we consider a superposition of Fock states that is truncated below a certain number of photons and optimize the NLSQ by selecting appropriate superposition coefficients [13]. Experimentally, we generate a superposition of a vacuum state and a single-photon state, and show that there is indeed a parameter that maximizes the NLSQ. The shapes of the wave packets of the generated states are tailored using a method similar to that of Ref. [19], so that we can implement real-time measurements. The amount of NLSQ observed here is higher than can be achieved with arbitrary Gaussian states, showing that the generated nonGaussian states are indeed appropriate approximations as ancillary states for the cubic phase gate. Note that there are already various demonstrations of generation of superposition of Fock states [21-28], where the superposition of up to three photons [28] has already been realized. However, the superpositions of Fock states demonstrated before are not sufficient to observe NLSQ, despite their nonclassical aspects as negative Wigner functions. On the other hand, this work shows the NLSQ of the superposition of a vacuum state and a single-photon state whose wave packet is compatible to real-time quadrature measurements.

This paper is structured as follows. Section II describes the concept of nonlinear squeezing and shows an explicit example of the cubic phase gate that is relevant to the experimental results. Section III explains the generation method of the superposition of the vacuum and the singlephoton states and how to tailor the temporal wave packet into an exponentially rising wave packet suitable for realtime measurements. Section IV explains the experimental setup. The experimental results are discussed in Sec. V, 
which shows the generated states and their NLSQ. Finally, Sec. VI gives a summary and future perspectives.

\section{NONLINEAR SQUEEZING}

We denote by $\hat{x}$ and $\hat{p}$ the quadrature operators whose commutation relation is $[\hat{x}, \hat{p}]=i$. We consider a type of operation called $N$ th-order phase gate, which is given by a unitary operator:

$$
\hat{U}_{N}(\kappa)=\exp \left(i \kappa \hat{x}^{N}\right)
$$

where $\kappa$ is a parameter that determines the strength of the operations. Note that this operator becomes displacement operation when $N=1$ and squeezing operation when $N=2$ [15]. When $N \geq 3$, the above operation is a nonGaussian operation that transforms Gaussian pure states to non-Gaussian ones. As $\hat{U}_{N}(\kappa)$ depends only on the operator $\hat{x}$, it leaves the operator $\hat{x}$ unchanged and transforms the operator $\hat{p}$ in the Heisenberg picture as

$$
\hat{p} \rightarrow \hat{p}+N \kappa \hat{x}^{N-1} .
$$

In the implementation of $\hat{U}_{N}(\kappa)$ using the measurementbased approach, the ideal ancillary states $\left|\psi_{\text {anc }}\right\rangle$ are given by [14]

$$
\left|\psi_{\text {anc }}\right\rangle=\hat{U}_{N}(\kappa)|p=0\rangle .
$$

In addition to the representation of the ancillary states using the state vectors, the above ideal ancillary states are also the zero eigenstates of the following operators:

$$
e^{i \kappa \hat{x}^{N}} \hat{p} e^{-i \kappa \hat{x}^{N}}=\hat{p}-N \kappa \hat{x}^{N-1} .
$$

For any realistic ancillary states, the variances of the operator in Eq. (4) will be nonzero. However, the smaller this variance is, the smaller the contribution of the ancillary state to the noises in the $N$ th-order phase gate becomes. As a concrete example, let us consider the implementation of the cubic phase gate in Fig. 1. The Heisenberg picture of the input-output relation of this circuit is given by [13]

$$
\begin{gathered}
\hat{x}_{\text {out }}=\frac{1}{\sqrt{2}} \hat{x}_{\text {in }}-\frac{1}{\sqrt{2}} \hat{x}_{\text {sqz }}, \\
\hat{p}_{\text {out }}=\sqrt{2}\left(\hat{p}_{\text {in }}+\frac{3 \kappa}{2 \sqrt{2}} \hat{x}_{\text {in }}^{2}\right)+\left(\hat{p}_{\text {anc }}-3 \kappa \hat{x}_{\text {anc }}^{2}\right) \\
+3 \gamma\left(\hat{x}_{\text {in }} \hat{x}_{\mathrm{sqz}}+\frac{1}{2} \hat{x}_{\text {sqz }}^{2}\right) .
\end{gathered}
$$

The first terms on the right-hand sides show the ideal cubic phase gate operations (with additional squeezing operations), while the second term of $\hat{p}_{\text {out }}$ is the nonlinear noise term due to the ancillary states. We observe that on the right-hand side of Eq. (6), the signs in front of $\hat{x}_{\text {in }}^{2}$ and $\hat{x}_{\text {anc }}^{2}$ are opposite. This is due to the difference between the active operations and the resource state needed. The amount of NLSQ in the ancillary states for the cubic phase gate will correspond to the noise contributions of this second term. On the other hand, the noises due to the terms concerning $\hat{x}_{\mathrm{sqz}}$ approach zero as the squeezing level of the ancillary squeezed state increases.

To define the NLSQ in an operational way that is experimentally useful, let us consider an approximation of the ancillary state $\left|\psi_{\mathrm{NL}}\right\rangle$ given by

$$
\left|\psi_{\mathrm{NL}}\right\rangle=\hat{S}(\lambda) \sum_{k=0}^{M} c_{k}|k\rangle,
$$

which is a superposition of Fock states up to $M$ photons, followed by the squeezing operations $\hat{S}(\lambda)$. The reason we consider the squeezing operation here is as follows. If we implement the squeezing operation and its Hermitian conjugate before and after $\hat{U}_{N}(\kappa)$, we can easily show that

$$
\hat{S}(\lambda) \hat{U}_{N}(\kappa) \hat{S}^{\dagger}(\lambda)=\hat{U}_{N}\left(\kappa / \lambda^{N}\right)
$$

where we assume that $\hat{S}^{\dagger}(\lambda) \hat{x} \hat{S}(\lambda)=\lambda \hat{x}$ and $\hat{S}^{\dagger}(\lambda) \hat{p} \hat{S}(\lambda)=$ $\hat{p} / \lambda$. As the squeezing operation is equivalent to simply adjusting the strength parameter $\kappa$, the optimal superposition coefficients $\left\{c_{k}\right\}$ are identical for all $\kappa$ and the optimal ancillary states of the different $\kappa$ are related simply via squeezing operations. This squeezing operation either can be implemented actively $[29,30]$ or can be compensated by adjusting the splitting ratios of the beam splitters in the circuit of Fig. 1. Therefore, without loss of generality, we assume that $\kappa=1$ and consider the cubic phase gate, i.e., the case where $N=3$ in Eq. (1), which is the case of our main interest. Then, we can define optimal nonlinear variance for any quantum state $\hat{\rho}$ as the minimum

$$
V_{\hat{\rho}}^{\mathrm{opt}}=\min _{\lambda>0}\left\{\operatorname{Tr}\left[(\Delta \hat{y})^{2} \hat{\rho}\right]\right\},
$$

where $\hat{y}=\lambda \hat{p}-3(\hat{x} / \lambda)^{2}, \Delta \hat{y}=\hat{y}-\operatorname{Tr}(\hat{y} \hat{\rho})$, and $\operatorname{Tr}(\cdot)$ is a trace of an operator. This optimization with respect to $\lambda>0$ guarantees that the optimal variances are the same for all the states that are related to each other via squeezing operations. We consider only positive $\lambda$ here as this preserves the form of the variances in Eq. (4), whereas negative $\lambda$ would represent squeezing and phase shift, but it would not lead to squeezed variance for the same state. To quantify how much a quantum state $\hat{\rho}$ is nonlinearly squeezed, we compare the amount of $\hat{V}_{\hat{\rho}}^{\text {opt }}$ to the smallest amount achievable with Gaussian states. Since Eq. (9) is invariant with respect to sign flip of $\hat{x}$, the optimal Gaussian state should be symmetric with respect to the $p$ axis. 
It can be also easily checked that the optimal displacement of $\hat{x}$ is zero and that displacement of $\hat{p}$ has no effect. On the other hand, the effect of squeezing is considered in the optimization with respect to $\lambda$. As a result, the optimal Gaussian state is the squeezed vacuum state and the Gaussian limit is given by $V_{|0\rangle\langle 0|}^{\mathrm{opt}}$ because the squeezing is included in the minimization over lambda. The quantum state is therefore nonlinearly squeezed when

$$
\frac{V_{\hat{\rho}}^{\mathrm{opt}}}{V_{|0\rangle\langle 0|}^{\mathrm{opt}}}<1
$$

and the amount of NLSQ is directly given by this ratio. For any state to be useful as an ancillary state, it must exhibit NLSQ as the vacuum state has a better noise performance as an ancillary state if NLSQ is not present. See Appendix A for more detailed calculations.

The simplest quantum state capable of exhibiting nonlinear squeezing can be prepared as a superposition of Fock states $c_{0}|0\rangle+c_{1}|1\rangle$. The parameters $c_{0}$ and $c_{1}$ can be optimized to obtain maximal relative reduction of variance $V_{\hat{\rho}}^{\mathrm{opt}} / V_{|0\rangle\langle 0|}^{\mathrm{opt}}$, which, for pure states, is obtained in state $0.79|0\rangle-0.61 i|1\rangle$ [13]. However, in the realistic scenario with losses, the optimal state can be different.

\section{GENERATION METHOD}

To generate the superposition of Fock states with an exponentially rising wave packet, we use an asymmetric optical parametric oscillator (OPO) [19], which is shown in Fig. 2. A superposition of the vacuum state and the singlephoton state is generated by a heralding scheme. Initially, a two-mode squeezed state

$$
|\psi\rangle=\sqrt{1-|q|^{2}}\left[|0\rangle_{s}|0\rangle_{i}+q|1\rangle_{s}|1\rangle_{i}+\mathcal{O}\left(|q|^{2}\right)\right]
$$

is generated from an $\mathrm{OPO}$, where $q$ is a parameter representing the strength of the two-mode squeezing, which we

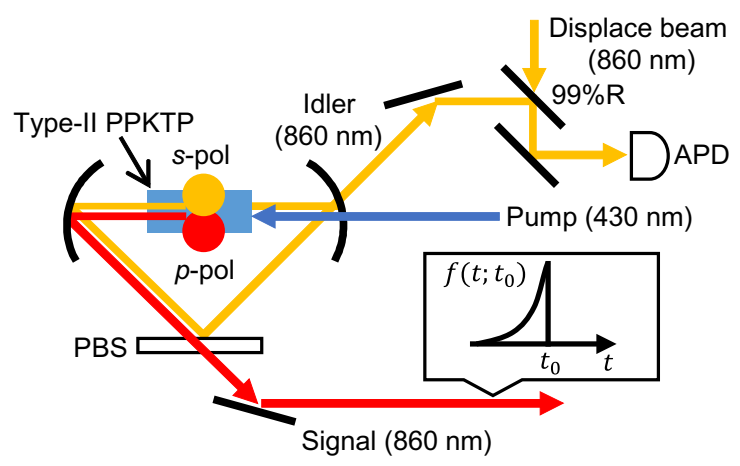

FIG. 2. Schematic diagram of the asymmetric optical parametric oscillator based on type-II PPKTP used in this experiment. PBS, polarization beam splitter; APD: avalanche photodiode. The displace beam is used in the preparation of the superposition between $|0\rangle$ and $|1\rangle$. assume to be sufficiently small, " $s$ " indicates signal mode, and " $i$ " indicates idler mode. These two modes are separated and we implement a weak displacement operation on the idler mode that results in

$$
\begin{aligned}
\hat{D}_{i}(\alpha)|\psi\rangle & =\exp \left(\alpha \hat{a}_{i}^{\dagger}-\alpha^{*} \hat{a}_{i}\right)|\psi\rangle \\
& \sim\left(|0\rangle_{s}-\alpha^{*} q|1\rangle_{s}\right)|0\rangle_{i}+\left(\alpha|0\rangle_{s}+q|1\rangle_{s}\right)|1\rangle_{i} \\
& \quad+\mathcal{O}\left[(|q|+|\alpha|)^{2}\right],
\end{aligned}
$$

where $\alpha$ represents the amount of the displacement and we assume $|\alpha| \ll 1$. Then, we consider the photon detection of the idler mode. When a photon number state $|1\rangle$ is detected, a superposition between $|0\rangle$ and $|1\rangle$ is induced in a signal mode:

$$
{ }_{i}\left\langle 1\left|\hat{D}_{i}(\alpha)\right| \psi\right\rangle \sim \alpha|0\rangle_{s}+q|1\rangle_{s} .
$$

The superposition coefficients are experimentally adjusted by changing the parameter $\alpha$ of the displacement operation and the strength $q$ of the two-mode squeezing.

In the asymmetric OPO used in this experiment, the signal mode and the idler mode have different polarization and one of the mirrors of the OPO is a polarization beam splitter. This OPO is designed so that the signal mode is nonresonant to the OPO. Ideally, the wave packet of the signal mode when the photon is detected at the idler mode is an exponentially rising shape [19]:

$$
f\left(t ; t_{0}\right) \propto e^{-\gamma\left|t-t_{0}\right| / 2} \Theta\left(t_{0}-t\right),
$$

where $\gamma$ is a parameter determined by the bandwidth of the $\mathrm{OPO}$ and $\Theta(\cdot)$ is a step function.

In the actual experiment, there are additional filtering cavities in the idler mode, which are used to remove unwanted frequency modes and they function as additional Lorenzian filters in the frequency domain. For the case where there are two filtering cavities, the shape of the wave packet when these filters are taken into account can be expressed as follows [19]:

$$
f\left(t ; t_{0}\right) \propto \sum_{n=1}^{3} c_{n} e^{-\gamma_{n}\left|t-t_{0}\right| / 2} \Theta\left(t_{0}-t\right),
$$

where $\gamma_{1}, \gamma_{2}$, and $\gamma_{3}$ correspond to the bandwidth of each cavity (OPO and the two filtering cavities), $c_{1}=1 /\left(\gamma_{2}-\right.$ $\left.\gamma_{1}\right)\left(\gamma_{3}-\gamma_{1}\right)$, and $c_{2}$ and $c_{3}$ are its cyclic permutations.

\section{EXPERIMENTAL SETUP}

Figure 3 shows the experimental setup. A continuous wave $(\mathrm{cw})$ titanium sapphire laser (MBR-110) operating at a wavelength of $860 \mathrm{~nm}$ is used as a light source of the experiment. Part of the laser beam is sent to a bow-tieshaped second harmonic generator and is converted to a 


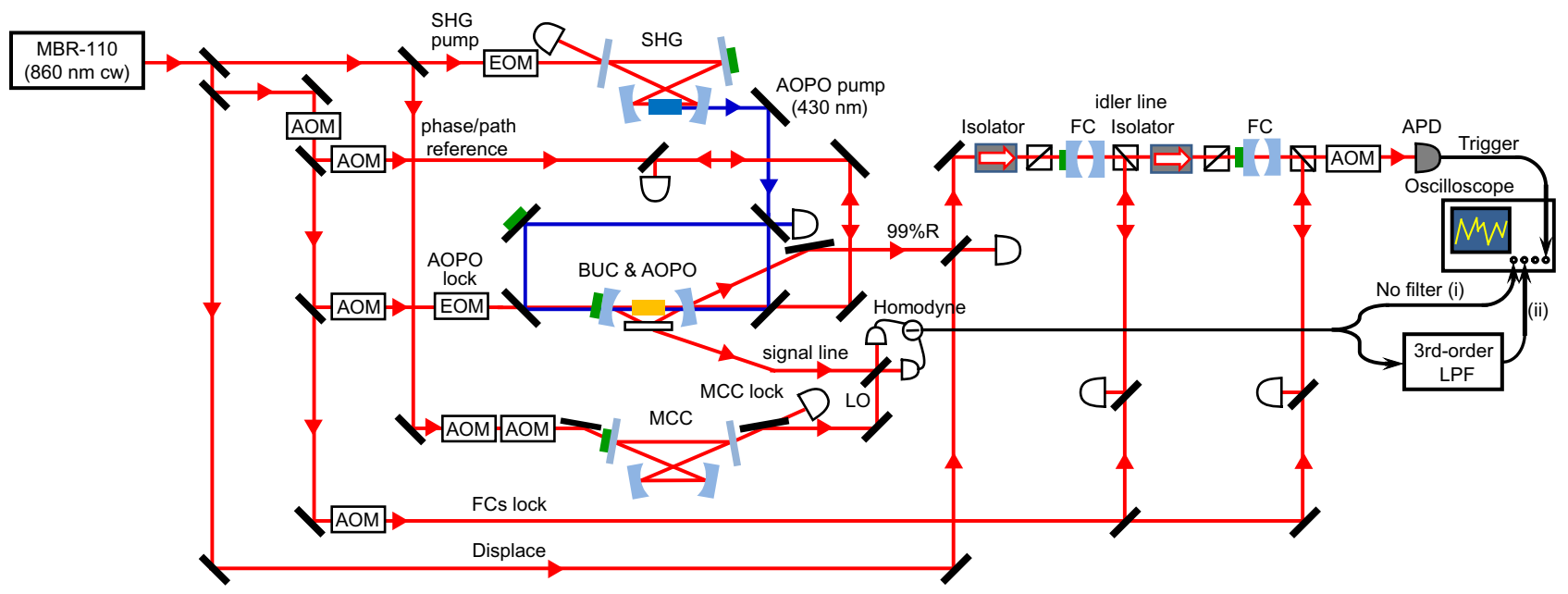

FIG. 3. Experimental setup for the generation of the superposition between $|0\rangle$ and $|1\rangle$. SHG, second harmonic generator; EOM, electro-optic modulator; AOM, acousto-optic modulator; AOPO, asymmetric optical parametric oscillator; BUC, buildup cavity; MCC, mode-cleaning cavity; FC, filter cavity; 99\%R, 99\% reflectivity; APD, avalanche photodiode; LO, local oscillator beam; LPF, low-pass filter.

$430 \mathrm{~nm}$ cw pump beam for the asymmetric OPO. In order to enhance the pump power and stabilize the optical path of the pump beam, a buildup cavity is constructed around the OPO. The asymmetric OPO is a triangle-shaped cavity with a linewidth of $33.7 \mathrm{MHz}$ at half-width half-maximum (HWHM) and a periodically poled $\mathrm{KTiOPO}_{4}$ crystal with type-II phase matching is placed inside the cavity. One of the mirrors of the OPO is a plate polarization beam splitter (PBS). The $s$-polarized idler mode is reflected by this PBS, and thus it is resonant to the OPO, while the $p$-polarized signal mode passes through the PBS and is off-resonant to the OPO. This results in the exponentially rising wave packet explained in Sec. III.

The displacement operation is implemented on the idler mode by interfering it with a coherent beam (displace beam) at a beam splitter with $99 \%$ reflectivity. The absolute value of the parameter $\alpha$ in Eq. (12) is adjusted by changing the power of this coherent beam and the phase of $\alpha$ is adjusted by the interference phase at this beam splitter. After the displacement operation, there are two filter cavities that remove unwanted frequency sideband modes. Both filtering cavities are Fabry-Perot cavities whose linewidths at HWHM are 140.1 and $90.9 \mathrm{MHz}$, respectively. The idler photons are then detected by an avalanche photodiode (APD) and the electrical signals from the APD are sent to an oscilloscope and used as the measurement triggers.

To stabilize the optical cavities and the relative phases at each beam splitter, lock beams and phase reference beams are employed. During state generations, however, as these beams might enter the APD and result in fake triggers, they are switched off using acousto-optic modulators. When these beams are turned off, the feedback controls are also off and the voltages of each feedback component are kept at the state immediately before. This method is called sample and hold and is widely used in the generation of non-Gaussian states [19,20,28,31].

To characterize the generated states, we perform homodyne measurements on the signal mode. A mode-cleaning cavity is used to spatially filter the local oscillator beam into a $\mathrm{TEM}_{00}$ mode and the power of the local oscillator beam is set to approximately $10 \mathrm{~mW}$. The homodyne detector has a flat frequency response up to approximately $200 \mathrm{MHz}$, which is much broader than the linewidth of the asymmetric OPO, meaning that the effects of the finite bandwidth of the homodyne detector are negligible.

The electrical signals from the homodyne detector are recorded by an oscilloscope. The sampling rate of the oscilloscope is $5 \mathrm{GS} / \mathrm{s}$ and the width of each frame is about $200 \mathrm{~ns}$, centered around the trigger signal of the APD. The electrical signals from the homodyne detector are split into two paths. While one of them is connected directly to the oscilloscope [signal (i) in Fig. 3] and is used in the digital postprocessing method, the other is filtered by a third-order low-pass filter whose time response function is designed to match the shape of the wave packet [signal (ii) in Fig. 3]. The electrically filtered signals are then recorded by the oscilloscope and these signals correspond to the quadrature values in the real-time measurement.

For each generated state, quadrature values in six measurement bases $\left(0^{\circ}, 30^{\circ}, 60^{\circ}, 90^{\circ}, 120^{\circ}\right.$, and $150^{\circ}$ from the axis of the $\hat{x}$ quadrature) with about 21000 events for each basis are measured. We reconstruct the density matrices of the generated states using the maximum likelihood method [32] from the obtained quadrature values of both the digital postprocessing method and the real-time measurement method. The NLSQ of each generated state is then calculated from the reconstructed density matrix. 


\section{EXPERIMENTAL RESULTS}

We generate superposition of a vacuum and a singlephoton state with nine different superposition coefficients. Note that the displacement beam is turned off when we generate the single-photon state. We use the single-photon states to first estimate the shape of the temporal mode function of the wave packet of the generated states. The estimation is done by using principal component analysis on the homodyne signals without the low-pass filter [signal (i) in Fig. 3] and the results are shown in Fig. 4. The estimated temporal mode function clearly exhibits the exponentially rising feature and its mode matching to the theoretically predicted mode is about $98 \%$. We also design a low-pass filter whose time response function matches with the temporal mode function. The time response function of the low-pass filter is shown as a red dashed curve in Fig. 4 and its mode matching to the temporal mode of the generated state is about $97 \%$, which is sufficient for real-time measurements.

Figure 5 shows the values of the nonlinear squeezing of the generated superpositions states and Wigner functions of some of the representative states. Note that the Wigner functions shown here are based on real-time measurements. In general, for the case of the pure state, the superposition of $|0\rangle$ and $|1\rangle$ can be parametrized as

$$
|\psi(\theta, \phi)\rangle=\cos \left(\frac{\theta}{2}\right)|0\rangle+e^{i \phi} \sin \left(\frac{\theta}{2}\right)|1\rangle
$$

Although the actual generated states are not pure states, the parameter $\theta$ is adjusted by looking at the ratio of the

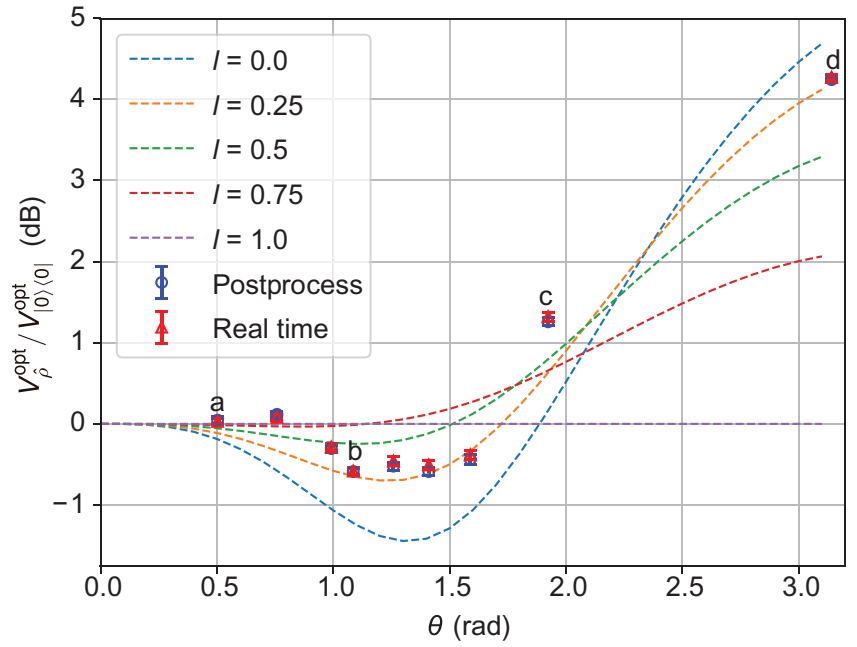

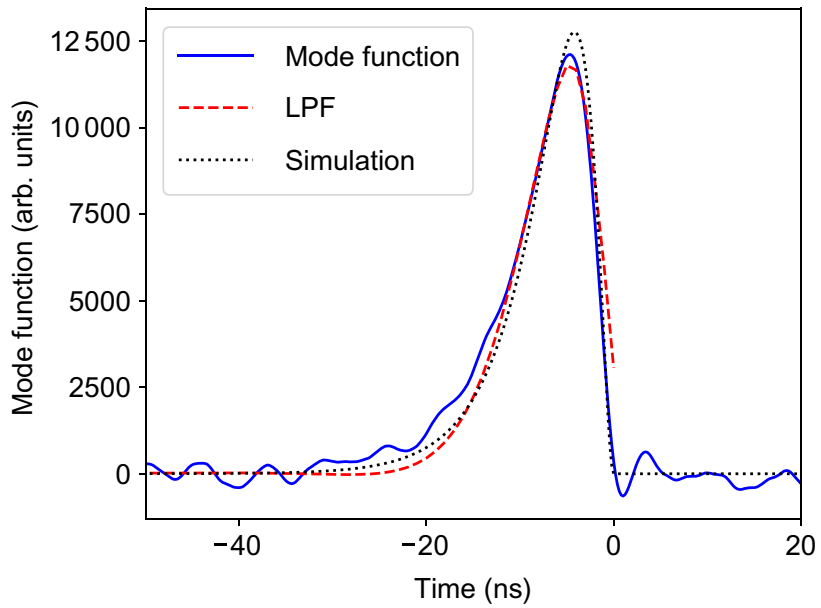

FIG. 4. Temporal mode function of the generated states. Blue solid curve: estimated temporal mode. Black dotted curve: temporal mode calculated by substituting the bandwidths of the cavities into Eq. (15). Red dashed curve: weighting function of the third-order low-pass filter for the real-time measurement.

count rate at the APD when the displacement beam is presented to when it is not presented, which corresponds to $\left[1+\tan ^{2}(\theta / 2)\right]: \tan ^{2}(\theta / 2)$ when the state is a pure state. The parameter $\theta$ in Fig. 5 is thus the parameter we adjust. On the other hand, the parameter $\phi$ is set to $\phi=3 \pi / 2$ by locking the reference phase of the beam splitter at the displacement operation.

From Fig. 5 we can see that not all parameters result in the superposition with NLSQ. One of the interesting features of NLSQ is that although the degree of the nonGaussianity of the quantum state is usually associated with
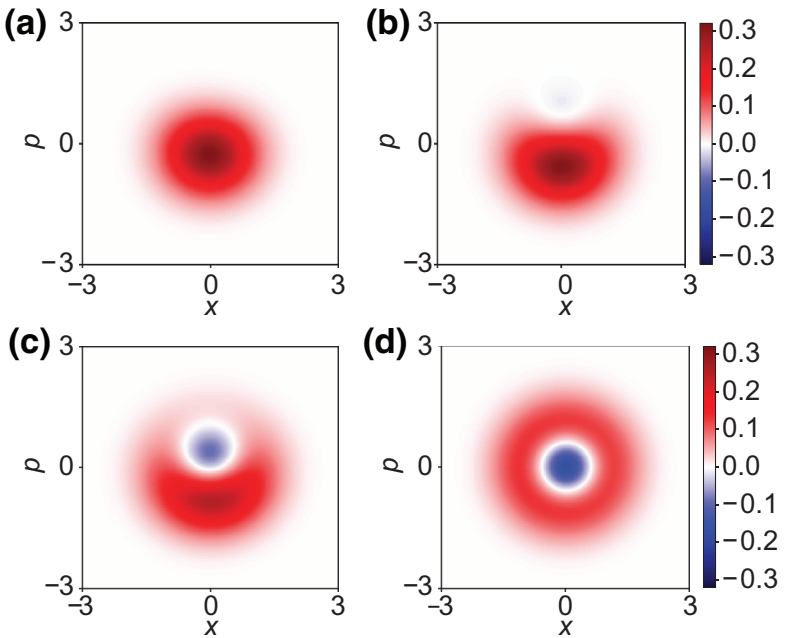

FIG. 5. NLSQ and some representative Wigner functions of the generated states. For the panel on the left, the blue circle markers and the red triangle markers correspond to the NLSQ of the states calculated using the digital postprocessing method and the real-time measurement method, respectively. The dashed lines are theoretical predictions when there are optical losses of $L$. Panels (a)-(d) on the right are the Wigner functions of the generated states that corresponded to each point of the plots on the left. Note that these are the Wigner functions for the real-time measurement method. 
how much the negative region of the Wigner function has [33], having large negative regions does not necessarily result in large NLSQ. For example, we can see that the single-photon state, a state with most negativity in our consideration, actually performs worst, with NLSQ of $4.24 \pm$ 0.03 and $4.27 \pm 0.03 \mathrm{~dB}$ for the digital postprocessing and the real-time methods, respectively. Therefore, in terms of noises of the cubic phase gate, the vacuum state performs better than the single-photon state. On the other hand, we observe clear NLSQ for the region where the parameter $\theta$ is between 1.0 and 1.6. For example, at $\theta=1.09 \mathrm{rad}$, which corresponds to point (b) in Fig. 5, the NLSQ values are $-0.59 \pm 0.04$ and $-0.58 \pm 0.04 \mathrm{~dB}$ for the digital postprocessing method and the real-time method, respectively. These values are better than the values that can be achieved with arbitrary Gaussian states (see also Appendix A). To also check whether the values of NLSQ of the generated states match with the experimental parameters or not, we also plot theoretical predictions for various optical losses $L$. From the plot, we can see that the experimental values of NLSQ can be relatively well explained by $L=0.25$, which is of the same order of magnitude as the experimental parameter. For further detailed discussions regarding the generated states, see Appendixes B and C. These results indicate that by making a non-Gaussian superposition, we can experimentally generate states with NLSQ that also have an exponentially rising wave packet appropriate for the implementation of the cubic phase gate in the time domain.

\section{SUMMARY AND OUTLOOK}

In summary, we have generated superposition between vacuum state and single-photon state with superposition coefficients such that it exhibits the NLSQ required for the cubic phase gate. Moreover, the generated states are in an exponentially rising wave packet, which allows us to use real-time quadrature measurements to evaluate the state. Therefore, the generated ancillary states are compatible with the currently promising time-domain-multiplexing method of optical CV quantum computation. Although the values of NLSQ are still relatively low, by increasing the number of photons and improving the optical losses of the system, we can expect that NLSQ can be much improved. Moreover, this idea of NLSQ is not limited to the cubic phase gate, but can be applied to higher-order phase gates as well [14]. Therefore, this work is a crucial step to extend the boundary of optical CV quantum information processing from the already widely researched Gaussian regime into the non-Gaussian regime.

\section{ACKNOWLEDGMENTS}

This work is partly supported by JSPS KAKENHI (Grants No. 18H05207, No. 18H01149, and No. 20K15187), the Core Research for Evolutional Science and Technology (CREST) (Grant No. JPMJCR15N5) of the Japan Science and Technology Agency (JST), UTokyo Foundation, and donations from Nichia Corporation. P.M. and R.F. acknowledge Grant No. GA18-21285S of the Czech Science Foundation and also support by national funding from MEYS and the European Union's Horizon 2020 (2014-2020) research and innovation framework program under Grant No. 731473 (project 8C20002 ShoQC). Project ShoQC has received funding from the QuantERA ERA-NET Cofund in Quantum Technologies implemented within the European Union's Horizon 2020 program.

\section{APPENDIX A: PARAMETERLESS NONLINEAR SQUEEZING}

Nonlinear squeezing of a quantum state can be fully characterized by the ratio $V_{\hat{\rho}}^{\mathrm{opt}} / V_{|0\rangle\langle 0|}^{\mathrm{opt}}$, where $V_{\hat{\rho}}^{\mathrm{opt}}=$ $\min _{\lambda>0}\left\{\operatorname{var}_{\hat{\rho}} \hat{y}\right\}$ with $\hat{y}=\lambda \hat{p}-3(\hat{x} / \lambda)^{2}$ and the variance is taken in state $\hat{\rho}$. Note that restricting $\lambda>0$ does not matter for the implementation of the quantum gate as it can be compensated by squeezing operations and the beam splitter. To prove this assertion, let us first show $V_{|0\rangle\langle 0|}^{\mathrm{opt}}$ is the minimal variance achievable for any Gaussian state:

$$
V_{\mathrm{Gauss}}^{\mathrm{opt}}=\min _{\hat{\rho} \in \mathcal{G}} \min _{\lambda>0} \operatorname{Tr}\left[(\Delta \hat{y})^{2} \hat{\rho}\right] .
$$

This follows from the nature of operator $\hat{y}$. Since it is symmetric with respect to $\hat{x}$, the optimal Gaussian state will be, up to a displacement that can be neglected, a squeezed vacuum state with quadrature operators $\hat{p}_{g}=g \hat{p}_{0}$ and $\hat{x}_{g}=\hat{x}_{0} / g$, where $\hat{x}_{0}$ and $\hat{p}_{0}$ are quadrature operators of the vacuum state. The minimal variance for a Gaussian state can then be rewritten as

$$
\min _{g>0} \min _{\lambda>0} \operatorname{Tr}\left\{\left[\Delta\left(g \lambda \hat{p}_{0}-3 \frac{\hat{x}_{0}^{2}}{g^{2} \lambda^{2}}\right)\right]^{2} \hat{\rho}\right\},
$$

and it can be immediately seen that $V_{\text {Gauss }}^{\mathrm{opt}}=V_{|0\rangle\langle 0|}^{\mathrm{opt}}$. The optimal Gaussian state is the vacuum state due to the flexibility in $\lambda>0$.

We can now define, for any single value of nonlinear parameter $\kappa$, the nonlinear variance as

$$
V_{\hat{\rho}}(\kappa)=\min _{\lambda>0} \operatorname{var}_{\hat{\rho}}\left(\lambda \hat{p}-\kappa \frac{\hat{x}^{2}}{\lambda^{2}}\right)
$$

and the relative nonlinear variance as

$$
R(\kappa)=\frac{V_{\hat{\rho}}(\kappa)}{V_{|0\rangle\langle 0|}^{\mathrm{opt}}(\kappa)} .
$$


By taking a different nonlinear parameter $\kappa^{\prime}=u^{3} \kappa$, we arrive at

$$
\begin{aligned}
V_{\hat{\rho}}\left(\kappa^{\prime}\right) & =\min _{\lambda>0} \operatorname{var}_{\hat{\rho}}\left(\lambda \hat{p}-\kappa u^{3} \frac{\hat{x}^{2}}{\lambda^{2}}\right) \\
& =u^{2} \min _{\lambda>0} \operatorname{var}_{\hat{\rho}}\left(\frac{\lambda}{u} \hat{p}-\kappa \frac{u^{2}}{\lambda^{2}} \hat{x}^{2}\right) \\
& =u^{2} V_{\hat{\rho}}(\kappa) .
\end{aligned}
$$

We can now see that $R(\kappa)$ does not actually depend on $\kappa$ and we can therefore choose it as we see fit.

\section{APPENDIX B: CORRELATIONS BETWEEN DIGITAL POSTPROCESSING AND REAL-TIME MEASUREMENTS}

In the main text, we show that the values of NLSQ are similar for the cases when the states are evaluated using both digital postprocessing and real-time measurements. However, to evaluate whether we have succeeded in the real-time measurements or not, we have to show that the quadrature values that are obtained via the real-time method are the same as those obtained with the postprocessing method for every single homodyne measurement.

As an example, Fig. 6 shows the quadrature distribution and the reconstructed Wigner functions of the target
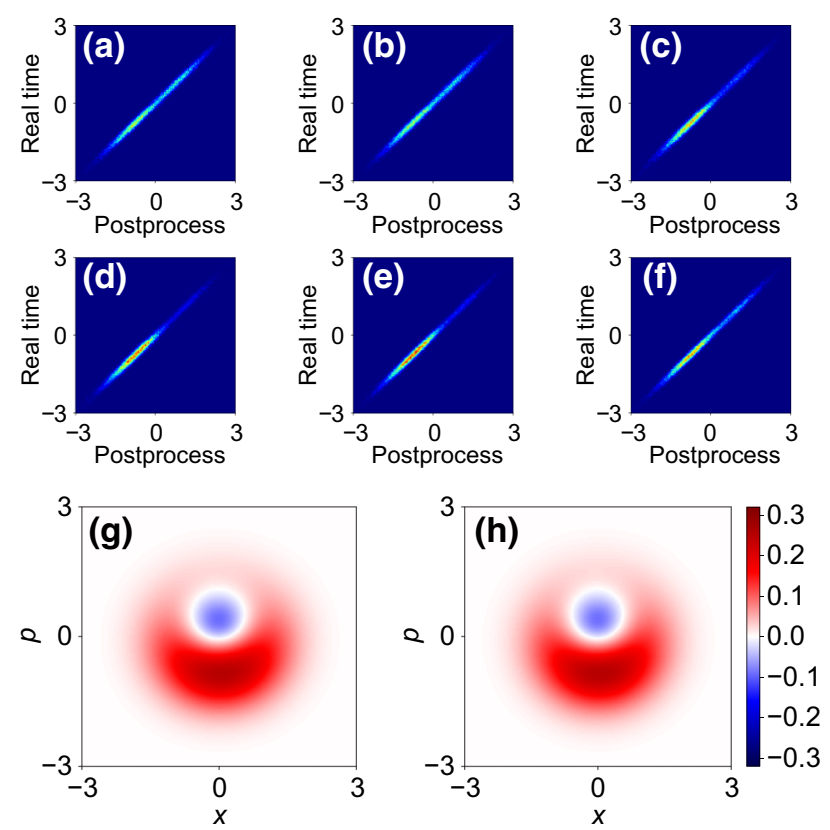

FIG. 6. Comparison between digital processing method and real-time method for point $\mathrm{c}$ in Fig. 5. (a)-(f) Correlations between quadrature values obtained via digital postprocessing and real-time methods for phases $0^{\circ}, 30^{\circ}, 60^{\circ}, 90^{\circ}, 120^{\circ}$, and $150^{\circ}$, respectively. $(\mathrm{g}),(\mathrm{h})$ Reconstructed Wigner functions using the quadrature values of the digital processing method and the real-time method, respectively. state that is equivalent to the Wigner function (c) of Fig. 5, analyzed with both the digital postprocessing and the realtime measurements. We observe that for any phase, the quadrature values of both methods are highly correlated with a correlation coefficient $>0.99$. The reconstructed Wigner functions are also qualitatively the same for both methods. For all the other superpositions, the situations are also similar and the correlation coefficients for all the generated states at all quadratures are $>0.98$. Therefore, we can conclude that we have succeeded in the real-time measurements of the superposition of the Fock states.

\section{APPENDIX C: DETAILED EVALUATIONS OF THE GENERATED STATES}

In the main text, we write the parametrized superposition between the vacuum state and the single-photon state as a pure state. However, the actual states are mixed states. If we let $L$ be the optical loss, the density matrix $\hat{\rho}(\theta, \phi, L)$ of the superposition can be written as

$\hat{\rho}(\theta, \phi, L)=\left(\begin{array}{cc}1-(1-L) \sin ^{2}\left(\frac{\theta}{2}\right) & \frac{1}{2} \sin (\theta) e^{-i \phi} \sqrt{1-L} \\ \frac{1}{2} \sin (\theta) e^{i \phi} \sqrt{1-L} & \sin ^{2}\left(\frac{\theta}{2}\right)(1-L)\end{array}\right)$.

As $\theta$ is the parameter we mainly adjust, we see whether the parameters $\phi$ and $L$ for each generated state are consistent over $\theta$ or not.

Figure 7 shows the fitting of $\phi$ and $L$ between the experimentally reconstructed density matrices and $\hat{\rho}(\theta, \phi, L)$ as a function of $\theta$. We observe that $\phi$ is close to $3 \pi / 2$ within a range of $\pm 0.2 \mathrm{rad}$. The only exception here is $\theta=\pi$, i.e., the single-photon state that is a phase-insensitive state, rendering the fitting inaccurate. On the other hand, the values of $L$ are mostly gathered around 0.25 for most values of $\theta$ except for small $\theta$. This behavior is expected as small $\theta$ means that most of the contribution of the state is due to the vacuum state, which is barely affected by losses, making the fitting of $L$ unreliable at a small $\theta$. (a)

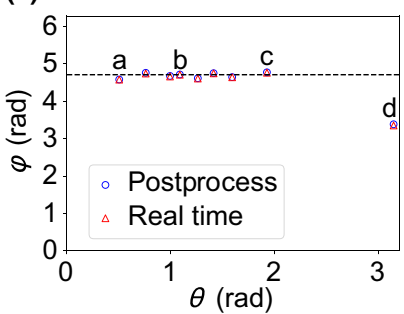

(b)

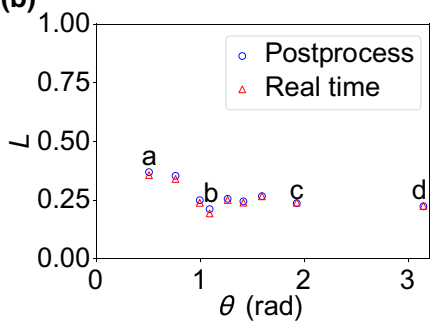

FIG. 7. Fitting of $\phi$ (a) and $L$ (b) of the generated states. Blue circle markers: digital postprocessing method. Red triangle markers: real-time measurement method. 
[1] R. Raussendorf and H. J. Briegel, A One-Way Quantum Computer, Phys. Rev. Lett. 86, 5188 (2001).

[2] N. C. Menicucci, P. van Loock, M. Gu, C. Weedbrook, T. C. Ralph, and M. A. Nielsen, Universal Quantum Computation with Continuous-Variable Cluster States, Phys. Rev. Lett. 97, 110501 (2006).

[3] S. Yokoyama, R. Ukai, S. C. Armstrong, C. Sornphiphatphong, T. Kaji, S. Suzuki, J. Yoshikawa, H. Yonezawa, N. C. Menicucci, and A. Furusawa, Ultra-large-scale continuous-variable cluster states multiplexed in the time domain, Nat. Photonics 7, 982 (2013).

[4] J. Yoshikawa, S. Yokoyama, T. Kaji, C. Sornphiphatphong, Y. Shiozawa, K. Makino, and A. Furusawa, Invited article: Generation of one-million-mode continuous-variable cluster state by unlimited time-domain multiplexing, APL Photonics 1, 060801 (2016).

[5] W. Asavanant, Y. Shiozawa, S. Yokoyama, B. Charoensombutamon, H. Emura, R. N. Alexander, S. Takeda, J. Yoshikawa, N. C. Menicucci, H. Yonezawa, and A. Furusawa, Generation of time-domain-multiplexed twodimensional cluster state, Science 366, 373 (2019).

[6] M. V. Larsen, X. Guo, C. R. Breum, J. S. NeergaardNielsen, and U. L. Andersen, Deterministic generation of a two-dimensional cluster state, Science 366, 369 (2019).

[7] W. Asavanant, B. Charoensombutamon, S. Yokoyama, T. Ebihara, T. Nakamura, R. N. Alexander, M. Endo, J. Yoshikawa, N. C. Menicucci, H. Yonezawa, and A. Furusawa, One-hundred step measurement-based quantum computation multiplexed in the time domain with $25 \mathrm{MHz}$ clock frequency, arXiv e-prints, arXiv:2006.11537 (2020).

[8] M. V. Larsen, X. Guo, C. R. Breum, J. S. NeergaardNielsen, and U. L. Andersen, Deterministic multi-mode gates on a scalable photonic quantum computing platform, arXiv e-prints, arXiv:2010.14422 (2020).

[9] S. Lloyd and S. L. Braunstein, Quantum Computation Over Continuous Variables, Phys. Rev. Lett. 82, 1784 (1999).

[10] S. D. Bartlett, B. C. Sanders, S. L. Braunstein, and K. Nemoto, Efficient Classical Simulation of Continuous Variable Quantum Information Processes, Phys. Rev. Lett. 88, 097904 (2002).

[11] D. Gottesman, A. Kitaev, and J. Preskill, Encoding a qubit in an oscillator, Phys. Rev. A 64, 012310 (2001).

[12] S. D. Bartlett and W. J. Munro, Quantum Teleportation of Optical Quantum Gates, Phys. Rev. Lett. 90, 117901 (2003).

[13] K. Miyata, H. Ogawa, P. Marek, R. Filip, H. Yonezawa, J. Yoshikawa, and A. Furusawa, Implementation of a quantum cubic gate by an adaptive non-gaussian measurement, Phys. Rev. A 93, 022301 (2016).

[14] P. Marek, R. Filip, H. Ogawa, A. Sakaguchi, S. Takeda, J. Yoshikawa, and A. Furusawa, General implementation of arbitrary nonlinear quadrature phase gates, Phys. Rev. A 97, 022329 (2018).

[15] K. Miyata, H. Ogawa, P. Marek, R. Filip, H. Yonezawa, J. Yoshikawa, and A. Furusawa, Experimental realization of a dynamic squeezing gate, Phys. Rev. A 90, 060302 (2014).

[16] A. Sakaguchi, H. Ogawa, M. Kobayashi, S. Suzuki, H. Yonezawa, E. Huntington, S. Takeda, J. Yoshikawa, and A. Furusawa, in 2018 Conference on Lasers and ElectroOptics (CLEO) (IEEE, San Jose, 2018), p. 1.

[17] B. W. Walshe, L. J. Mensen, B. Q. Baragiola, and N. C. Menicucci, Robust fault tolerance for continuous-variable cluster states with excess antisqueezing, Phys. Rev. A 100, 010301 (2019).

[18] N. C. Menicucci, Temporal-mode continuous-variable cluster states using linear optics, Phys. Rev. A 83, 062314 (2011).

[19] H. Ogawa, H. Ohdan, K. Miyata, M. Taguchi, K. Makino, H. Yonezawa, J. Yoshikawa, and A. Furusawa, Real-Time Quadrature Measurement of a Single-Photon Wave Packet with Continuous Temporal-Mode Matching, Phys. Rev. Lett. 116, 233602 (2016).

[20] W. Asavanant, K. Nakashima, Y. Shiozawa, J. Yoshikawa, and A. Furusawa, Generation of highly pure Schrödinger's cat states and real-time quadrature measurements via optical filtering, Opt. Express 25, 32227 (2017).

[21] A. I. Lvovsky and J. Mlynek, Quantum-Optical Catalysis: Generating Nonclassical States of Light by Means of Linear Optics, Phys. Rev. Lett. 88, 250401 (2002).

[22] K. J. Resch, J. S. Lundeen, and A. M. Steinberg, Quantum State Preparation and Conditional Coherence, Phys. Rev. Lett. 88, 113601 (2002).

[23] C. H. H. Schulte, J. Hansom, A. E. Jones, C. Matthiesen, C. Le Gall, and M. Atatüre, Quadrature squeezed photons from a two-level system, Nature 525, 222 (2015).

[24] Y. Hashimoto, T. Toyama, J. Yoshikawa, K. Makino, F. Okamoto, R. Sakakibara, S. Takeda, P. van Loock, and A. Furusawa, All-Optical Storage of Phase-Sensitive Quantum States of Light, Phys. Rev. Lett. 123, 113603 (2019).

[25] E. Bimbard, N. Jain, A. MacRae, and A. I. Lvovsky, Quantum-optical state engineering up to the two-photon level, Nat. Photonics 4, 243 (2010).

[26] J. C. Loredo, C. Antón, B. Reznychenko, P. Hilaire, A. Harouri, C. Millet, H. Ollivier, N. Somaschi, L. De Santis, A. Lemaître, I. Sagnes, L. Lanco, A. Auffèves, O. Krebs, and P. Senellart, Generation of non-classical light in a photon-number superposition, Nat. Photonics 13, 803 (2019).

[27] T. J. Bartley, G. Donati, J. B. Spring, X.-M. Jin, M. Barbieri, A. Datta, B. J. Smith, and I. A. Walmsley, Multiphoton state engineering by heralded interference between single photons and coherent states, Phys. Rev. A 86, 043820 (2012).

[28] M. Yukawa, K. Miyata, T. Mizuta, H. Yonezawa, P. Marek, R. Filip, and A. Furusawa, Generating superposition of up-to three photons for continuous variable quantum information processing, Opt. Express 21, 5529 (2013).

[29] R. Filip, P. Marek, and U. L. Andersen, Measurementinduced continuous-variable quantum interactions, Phys. Rev. A 71, 042308 (2005).

[30] Y. Miwa, J. Yoshikawa, N. Iwata, M. Endo, P. Marek, R. Filip, P. van Loock, and A. Furusawa, Exploring a new Regime for Processing Optical Qubits: Squeezing and Unsqueezing Single Photons, Phys. Rev. Lett. 113, 013601 (2014).

[31] K. Wakui, H. Takahashi, A. Furusawa, and M. Sasaki, Photon subtracted squeezed states generated with periodically poled ktiopo4, Opt. Express 15, 3568 (2007).

[32] A. I. Lvovsky, Iterative maximum-likelihood reconstruction in quantum homodyne tomography, J. Opt. B: Quantum Semiclassical Opt. 6, S556 (2004).

[33] A. Kenfack and K. yczkowski, Negativity of the wigner function as an indicator of non-classicality, J. Opt. B: Quantum Semiclassical Opt. 6, 396 (2004). 